\title{
Cristina Lindenmeyer
}

\section{Etre en suspens}

Mon propos est focalisé sur la médecine actuelle celle qui traite le sujet de façon de plus en plus anonyme et dont la démarche semble être de laisser de côté, voire négliger, ce qui nous est le plus intime - notre rapport à notre propre corps, - pouvant ainsi déclencher des processus subjectifs des plus complexes. Entre les sujets en position de demande et le médecin qui s'arroge une position supposée de savoir, circulent des fantasmes les plus fous.

Nous savons que l'une des caractéristiques du cancer est d'apparaître à tout moment dans l'organisme humain sans que le malade, du moins au cours des premières phases de la maladie, ne ressente la moindre douleur.

Les techniques actuelles, particulièrement développées dans les hôpitaux spécialisés dans le traitement du cancer, permettent aux médecins de détecter le développement de la maladie, grâce à diverses investigations techniques très au point et avant même que le malade ait pu percevoir la moindre modification corporelle. Ces techniques médicales offrent la possibilité d'accéder aux lieux les plus profonds du corps humain : son organisation cellulaire.

C'est donc le savoir médical qui détecte la présence de la maladie, sans que le patient en ait nécessairement été alerté de quelque manière que soit. Autrement dit, c'est l'intervention du médecin qui va déclencher chez le patient la prise de conscience qu'il souffre d'une maladie grave et qu'il se trouve menacé de mort.

C'est dire que la situation de maladie, telle qu'elle est vécue par nos patients, n'apparaît pas comme l'effet d'un trauma qui aurait déjà eu lieu mais comme le trauma lui-même. L'effet traumatique de l'annonce de la maladie potentiellement mortelle provoque une certaine perception corpo-

Cristina Lindenmeyer, psychanalyste, maître de conférences à l'Université Paris 7-Denis-Diderot, 44 rue de Sévigné, 75003 Paris. 
relle à partir de laquelle on assiste alors à la recomposition, chez le patient, de son fantasme de mort et ce nouvel assemblage définit les conditions nécessaires à la réactualisation des fantasmes infantiles.

Mon expérience de psychothérapeute auprès de ces patients, à partir du moment où ils deviennent patients avant même de se sentir malade, m'a montré que le corps se constitue comme un objet phobogène, lieu de projection de l'infantile. Il en résulte souvent que le patient adopte un comportement d'évitement de caractère souvent phobique : le corps fait soudain peur, devient complètement étranger ou un élément étranger.

Cette « étrangeté » du corps me semble être accentuée par une sorte de reprise active du traumatique : quelque chose paraît maîtrisable sous la forme d'une théorisation - comme nous la voyons à l'œuvre dans les théories sexuelles infantiles.

Nous pouvons parler alors de cette reprise de son histoire sous forme d'un « roman », comme le souligne M.J. Del Volgo ${ }^{1}$, plus proche d'un récit événementiel que d'un véritable processus de subjectivation.

Sous l'effet du choc, la maladie réveille quelque chose en soi en attente d'un dire. La réalité de la souffrance vécue par l'intermédiaire du corps semble transformer la réalité présente (celle du cancer) en la réactualisant, sous forme d'une menace de mort archaïque. Comme le rappelle P. Fédida dans son texte " L'hypocondriaque médecin ${ }^{2}$ », ces personnes paraissent être à la recherche d'une maladie comme façon de négocier avec une angoisse archaïque. Cette angoisse trouvera un lieu d'expression à travers la maladie.

C'est alors, soit d'une attitude extrêmement défensive contre l'irreprésentable de la mort, ce qui précipiterait le sujet dans une sorte de contrainte ultime à répéter, soit, au contraire, une attitude n'entraînant aucune résistance à la mort (mort réelle, mort dans le réel).

L'énoncé du diagnostic de la maladie va provoquer une coupure entre un état antérieur de confiance en soi, où la capacité de faire face au monde était assurée, et un état de désillusion et l'effondrement de l'illusion d'un pouvoir en fonction duquel rien ne pouvait arriver. Cette désillusion va se traduire par une plainte souvent entendue chez ces patients : "Je ne pouvais pas croire qu'un jour ça pourrait m'arriver à moi. »

Nous pouvons donc parler de la découverte de la maladie comme d'une expérience qui fait régresser le sujet à une situation primitive de détresse où il n'a plus les moyens psychiques d'élaborer. L'expérience de nos patients

1. M. J. Del Volgo, «L'instant de dire », dans Cliniques méditerranéennes, n 37-38, 1993, p. 63-77.

2. P. Fédida, "L'hypocondriaque médecin », dans Monographie de la revue française de psychanalyse, L'hypocondrie, Paris, p.113-136. 
cancéreux s'avère donc en de nombreux points comparables à la situation de l'enfant démuni face à la toute puissance de l'adulte dont il dépend.

$\mathrm{Du}$ point de vue de la réalité psychique, cette situation de détresse induite par la situation de maladie va être vécue comme une répétition. Répétition d'une scène primitive d'absence de protection de celle qui aurait dû le protéger.

Tout se passe alors comme si les échanges, les transmissions du corps au sens propre passaient par les mains de la mère ou de ses substituts (la mère réelle, l'infirmière, l'équipe médicale en général), mais comme une mère instable à laquelle on ne peut pas faire confiance, une mère qui abandonne. Moments, dans le réel de la maladie, qui vont permettre au sujet de « condenser en un seul bloc » ses expériences traumatiques.

Paradoxalement, le traitement médical se présentera comme la seule façon de lutter contre le cancer et qui pourra désormais expulser ses sensations d'un corps contaminé. Le traitement chimiothérapeutique apparaît paradoxalement comme l'unique moyen de stabiliser le corps envahi.

Il sera même possible de considérer les sensations éprouvées en raison du traitement comme la reviviscence de sensations corporelles (être transporté d'un lieu à l'autre, fièvre, vomissements, menaces de vertige).

\section{L'IDENTIFICATION À L'AGRESSEUR}

En énonçant le diagnostic, le médecin ne « crée » pas seulement la maladie, il en propose aussi la guérison, devenant par là même l'unique recours capable d'annuler cette expérience insupportable, d'où la construction d'une alliance qui, par bien de ses aspects, fait penser à une servitude. Ce que nos patients confirment volontiers en parlant de soumission à leur médecin.

Philippe s'adresse à moi, alors qu'il doit à nouveau subir une chimiothérapie et éprouve une sensation diffuse de mal-être qu'il ne peut que mettre en rapport avec la nouvelle de sa maladie.

Après son premier cancer tout allait bien, trop bien peut-être. Jusqu'au jour où à nouveau le médecin lui annonce l'apparition d'une nouvelle tumeur cancéreuse.

Tout au long des premières séances il me raconte son histoire sous la forme d'un discours sans fin, qui ne débouche sur rien. Il m'explique qu'il ne peut pas, dans ces conditions, associer quoi que soit sur ce que son cancer vient raconter. Le symptôme somatique prend ici tout son poids, et nous semble primordial pour comprendre comment il est vécu par le sujet.

Les silences obstinés des séances font enfin surgir un souvenir qui deviendra répétitif : « un jour, à la plage, alors que j'étais petit, je me suis fait mal au genou. Je saignais beaucoup, et ma mère m'a amené à un endroit, là- 
bas, où il y avait des pompiers avec des trucs de secours. Il y avait des seringues et des produits. Quand j'ai regardé dehors il y avait un homme, pas un médecin, un homme habillé avec des habits de travail. Il avait une scie. Quand il est passé, il m'a montré la scie et m'a dit : je vais te couper ton genou. »

Cette « complaisance somatique ${ }^{3}$ » permet de comprendre comment le corps peut servir d'écran projectif sur lequel se révèle l'image du passé. Pendant les séances suivantes, Philippe va revenir plusieurs fois sur ce souvenir dont les éléments sont comme des « restes diurnes » dans le rêve. Des « restes diurnes » qui, comme dans le rêve, fonctionnent aussi par une connivence avec la langue : couper le genou est l'acte castrateur redouté que le médecin pourrait exécuter sur son corps.

Les sensations du corps malade vont servir de support au surgissement de ses histoires infantiles. Les choses se passent comme si cette situation de maladie pouvait entraîner inévitablement l'émergence d'un sexuel traumatique qui déborde les moyens symboliques du sujet.

D'après nos observations, nos patients établissent avec leur médecin un rapport infantile et deviennent des enfants vulnérables, fragiles, mais aussi un enfant séduit. Et ce sera cette fragilité qui les amènera dans le fantasme, à des aménagements pulsionnels où s'installera le sadisme de l'adulte séducteur. Le médecin en vient à être le séducteur puisqu'au moment où il annonce la maladie, il propose aussi la guérison, en se maintenant dans une position prophétique.

Ainsi devient-il le lieu où les intrications de la libido et de la pulsion de mort apparaissent à travers des moments sadiques, liés aux mutilations du corps et à sa décomposition.

S'installe alors un jeu terrifiant entre le pouvoir du médecin et le pouvoir de la mort. Dans les cas de rechute et reprise du traitement, la force se trouve du côté de la mort, figure confondue avec le médecin devenu persécuteur à travers le corps, sans cesse castré.

Nous savons que, pour Ferenczi, ce qui fait trauma dans l'acte de séduction, n'est pas autant le contenu de l'acte, mais la différence du langage sexuel entre le séducteur (l'adulte) et le séduit (l'enfant). C'est la méconnaissance du langage sexuel de l'adulte qui introduit cette dimension traumatique pour l'enfant. En étant l'adulte qui établit les règles, son autorité alimente son pouvoir de séducteur.

3. M.J. Del Volgo, R. Gori, Y. Poinso, « Roman de la maladie et travail de formation du symptôme. Complémentarité des approches psychanalytique et médico-biologique », dans Psychologie médicale, 26, 14, 1994, 1434-1438. 
Ferenczi insiste sur cet état de détresse de l'enfant face à l'adulte qui impose l'acte. Le temps de l'effroi se situe au moment où l'adulte, au lieu de fonctionner comme " pare-excitation », devient celui qui excite, plongeant l'enfant dans un mouvement pulsionnel sans retenue.

Cette double fonction du médecin, annoncer la maladie et proposer sa guérison, le fait entrer dans la catégorie de mauvais parent : au lieu de fonctionner comme pare-excitation par son rôle de soignant, il devient mauvais, puisque incapable d'arrêter les attaques de la mort.

En soulignant l'importance de l'efficacité thérapeutique, le discours médical met l'accent sur la technique et occulte la nécessité de l'accueil de la vie fantasmatique du sujet.

Tant que le patient se maintiendra dans une extériorité par rapport à son corps libidinal, facilitant ainsi la parole médicale, il restera hypnotisé par l'agresseur, et répétera un comportement infantile, de soumission aux mauvais parents. Tel serait le noyau d'une position sadomasochiste. Dépossédé de son propre corps, le patient témoigne d'aménagements pulsionnels les plus variés, débouchant sur les formes les plus morbides.

\section{MISE EN SUSPENSION}

Revenons aux élaborations de Ferenczi à propos des réactions possibles au traumatisme : une des réactions pourrait se caractériser comme une espèce d'éclatement pouvant aller jusqu'à une totale atomisation de soimême, atomisation qui serait la seule forme possible de survie. L'instauration d'un clivage narcissique de sauvetage peut alors s'avérer être la solution au trauma.

Ce clivage narcissique peut provoquer un mode d'autoguérison qui va permettre à un sujet narcissiquement blessé de faire face à une situation insoutenable. Ce mode de fonctionnement permet au sujet de se maintenir dans l'illusion d'un entourage protecteur face à la force dévastatrice de la situation.

Cette question d'un aménagement psychique face au trauma devient donc la matrice d'une organisation où l'enfant (l'enfant chez l'adulte) se retrouve écarté de lui-même : il ne peut alors qu'observer la situation dans un clivage psychique qui témoigne de son intense douleur interne.

Cette position de secours à soi-même renvoie non seulement à l'idée d'identification à l'agresseur mais aussi à celle d'une relation narcissique comme moyen de sauvetage, position importante pour comprendre la relation d'objet impossible.

Ce qui, si nous essayons de réfléchir sur le plan corporel, est très important. À ce moment où l'on se retrouve « délivré de la pesanteur terrestre » 
c'est toute une possibilité d'habiter le corps qui se présente, mais de l'habiter en suspension. Comme si le corps était certes là, mais le sujet du corps ailleurs, dans l'air, suspendu. Cette référence à ce lieu de suspension peut aisément se traduire dans la situation clinique par des moments où le sujet parle de son corps comme un objet détaché, comme s'il ne lui appartenait pas, dans une sorte de dédoublement de soi.

Ce qu'il faut souligner encore ici, c'est la nécessité d'être à distance, mais dans une distance qui évoque les hauteurs comme si de s'imaginer flotter dans le ciel constituait le confort par excellence. Sortir de son propre corps ou de son propre psychique, telle est la destinée souhaitée. C'est donc par le clivage - lui-même issu de cette suspension - qu'une partie du sujet va pouvoir s'occuper de l'autre partie, comme le fait un bon parent.

Dans cette quête pour échapper à l'insupportable, le sujet fait de son corps le lieu de la bataille. Lieu choisi pour permettre au psychique de se mettre à distance, en même temps que le corps devient cette scène où la bataille va pouvoir être montrée à l'entourage et constituer ainsi un appel au secours.

On peut trouver certains points communs entre ce que nous décrit Ferenczi et ce que certains de nos patients peuvent exprimer : l'impression, à partir du moment où le diagnostic leur a été énoncé, de ne plus habiter son propre corps. Comme si, dès lors, tout était paralysé, bloqué, congelé, et ne pouvant plus être réinvesti. L'inscription de cette expérience d'effroi et d'effraction, se recouvre d'une masse rigide d'évitement et de précautions, dans la période qui suit immédiatement le choc, stratégie d'évitement qui se traduit généralement par un épuisement du travail psychique mobilisé dans une visée d'autoprotection. Cette sensation se présente quelquefois au cours de la séance comme si tout à coup le corps devenait habitable, mais avec la lourdeur d'une histoire qui déborde les moyens disponibles pour l'enfant en soi pour l'élaborer. Certains patients éprouvent - au cours de la situation analytique - le sentiment, la sensation de ne plus être invisible, d'autres encore ressentent la situation comme un trou dans lequel ils sont susceptibles de tomber, une tentative $d^{\prime}$ annuler cette lourdeur, ou la sensation de vivre dans cette sorte de suspension.

Les séances peuvent parfois être vécues comme une opportunité de s'appuyer sur l'autre, comme une sorte de relaxation de cette pesanteur du corps sur l'autre qui le soutient. Comme s'il y avait recherche d'un lieu, ou d'un temps où le sujet pouvait se sentir soutenu. C'est par exemple, le cas des patients qui se comportent comme si l'analyste était un réceptacle pour leurs paroles. Ils enchaînent d'une séance à l'autre comme s'ils n'avaient pas besoin de reprendre ce qui avait été dit à la séance précédente. 
Il m'est ainsi plusieurs fois arrivé que mes patients reprennent les séances de psychothérapie, après l'interruption des vacances, dans le droit fil de ce qui avait été dit à la séance précédente, comme s'il n'y avait eu aucune rupture, comme si le sentiment de continuité ne pouvait pas être perturbé. Ce qui leur importe, dans le même temps, c'est de rester dans l'illusion d'être complètement contenus par l'autre, porté par l'autre. Comme si, en étant porté par l'autre, on ne pouvait ni chuter, ni rechuter.

\section{CONCLUSION}

Lorsque les représentations médicales s'avèrent incapables d'aider les patients à élaborer leur état de maladie, les médecins les adressent au psychanalyste.

Ce que le travail analytique peut réintroduire, c'est précisément, l'élaboration de cette dimension traumatique et angoissante qui s'impose à eux, à l'occasion de cette confrontation avec la menace de mort qu'introduit le cancer. Son rôle sera de permettre une perlaboration, au sens freudien $d u$ Durcharbeiten, de ces éléments du passé, à travers le travail dans le transfert.

Ce qui veut dire que le travail de l'analyste est loin d'être aisé. Non seulement il peut être mis en position d'acteur d'une violence exercée à l'encontre du sujet, mais aussi mis au défi dans ses capacités à contenir. $\mathrm{N}^{\prime}$ oublions pas que la violence n'est pas seulement impossibilité de subjectivation, mais aussi cette sorte d'absence/présence nécessaire pour faire face à la détresse au moment même où il devient impératif qu'un autre vienne nous prêter secours.

Combien de fois me suis-je sentie pétrifiée, paralysée dans ma possibilité de penser, comme si tout mon corps se retrouvait contraint à ne plus faire le moindre geste, à ne plus pouvoir bouger. Dans une mortification qui faisait naître chez moi le désir, par exemple, d'arrêter avant l'heure les séances, de ne plus entendre ce qui m'était dit, ou encore, de rester dans une vigilance méticuleuse - enfin une série de situations qui visait à faire ressentir chez l'autre la souffrance qui se trouve en soi.

Ce qui nous permet d'avancer que l'idée de maladie recèle aujourd'hui cette particularité : celle d'être malade en occultant l'expérience de la maladie. Expérience de maladie niée, " mise en suspension », dirais-je, et qui n'est plus ce moment d'expression d'un monde interne, mais bien plutôt un destin vidé de sens. Tout se passe comme si le sujet voulait faire expérimenter à l'analyste une situation de détresse, et tester dans le même temps si celui-ci peut en faire quelque chose. 
Comme si ce qui se trouve en jeu est la possibilité d'une constitution même du narcissisme. Comme s'il s'agissait d'un dédoublement qui permettra la reconnaissance de l'altérité.

\section{BIBLIOGRAPHIE}

BRUN, D. 2001. L'enfant donné pour mort : la pire des choses qui puisse arriver à une femme, Paris, Éd. Eshel.

CAnguilhem, G. 1994. Le normal et le pathologique, 5e éd., Paris, Puf.

Del Volgo, M.-J. ; Gori, R. ; Poinso, Y. 1994. « Roman de la maladie et travail de formation du symptôme. Complémentarité des approches psychanalytique et médico-biologique », dans Psychologie médicale, 26, 14, 1434-1438.

Del Volgo, M.-J. 1997. L'instant de dire, Toulouse, érès.

Del Volgo, M.-J. ; Gori, R. 2005. La santé totalitaire. Essai sur la médicalisation de l'existence, Paris, Denoël.

FEDIDA, P. 1992. Crise et contre-transfert, Paris, PUF, 1995.

FedidA, P. «L'hypocondriaque médecin », L'hypocondrie, dans Monographie de la revue française de psychanalyse, Paris, 113-136.

FerencZi, S. 1933. « Confusion de langue entre les adultes et l'enfant. Le langage de la tendresse et le langage de la passion », dans CEuvres complètes, vol. IV, Paris, Payot, 1982 (art. posthumes).

FERENCZI, S. « Réflexions sur le traumatisme », dans CEuvres complètes, vol. IV, Paris, Payot.

Foucault, M. 1993. Naissance de la clinique, $3^{e}$ éd., Paris, Puf.

Freud, S. 1915. « Pulsions et destins de pulsions », dans CEuvres complètes, vol. XIII, Paris, PUF, 1994.

FREUD, S. 1919-1920. «Au-delà du principe de plaisir », dans CEuvres complètes, vol. XV, Paris, PUF, 1996.

FREUD, S. 1925. " Inhibition, symptôme et angoisse », dans CEuvres complètes, vol. XVII, Paris, PUF, 1992.

GORI, R. 1996. La preuve par la parole, Paris, PUF.

KHAN, M. 1979. Figures de la perversion, Paris, Gallimard.

McDougall, J. 1989. Théâtres du corps, Paris, Gallimard.

McDougall, J. 1998. Eros aux mille et un visages, Paris, Gallimard.

WinnicotT, D.W. 1975. Jeu et réalité. L'espace potentiel, Paris, Gallimard.

Résumé

Cet article essaie de montrer l'intérêt de traiter la problématique de l'effet du diagnostic du cancer, suite à la mise en place de techniques de détection précoce de cette maladie. Il en ressort une spécification de l'approche clinique psychanalytique, où l'auteur introduit une réflexion à partir des articles de Ferenczi.

Le recours à ces concepts soutient la réflexion de l'auteur sur la complexité transférentielle de la prise en charge du patient cancéreux. 
Mots clés

Cancer, méthodes modernes de détection, traumatisme, identification à l'agresseur.

THE WAITING BEING

\section{Summary}

This article regards the problems that the diagnostic of a cancer may produce on people undergoing early detection techniques for this disease. The specificity of this psychoanalytic clinical work is described and to which the author adds her reflections in accordance with Ferenczi's later publications which also contribute to the depth of her clinical experience.

These concepts support the author's reflection upon the transferential complexity of work with cancer patients.

\section{Keywords}

Cancer, modern methods of detectim, trauma, identification to aggressor. 